\title{
ROLE OF ELECTROPHYSIOLOGICAL STUDY AND CATHETER ABLATION FOR RECURRENT VENTRICULAR TACHYCARDIA COMPLICATING MYOCARDITIS
}

\author{
Emanuele Cecchi, Serena Fatucchi, Elena Crudeli, Cristina Giglioli
}

\author{
Department of Heart and Vessels, Azienda Ospedaliero-Universitaria Careggi, Florence, Italy
}

\begin{abstract}
Summary: Here we report the case of a 31-year-old man admitted to our hospital with echocardiografic and Cardiac Magnetic Resonance signs of myocarditis complicated by ventricular tachycardia, initially resolved with direct current shock. After the recurrence of ventricular tachycardia the patient was submitted to electrophysiological study revealing a re-entrant circuit at the level of the medium segment of interventricular septum, successfully treated with transcatheter ablation. This case highlights how the presence of recurrent ventricular arrhythmias at the onset of acute myocarditis, suspected or proven, could be associated with a pre-existing arrhythmogenic substrate, therefore these patients should be submitted to electrophysiological study in order to rule out the presence of arrhythmogenic focuses that can be treated with transcatheter ablation.
\end{abstract}

Key words: Myocarditis; Ventricular arrhythmias; Electrophysiological study; Catheter ablation

\section{Introduction}

The clinical presentation of myocarditis may include a wide variety of signs and symptoms from subclinical disease to acute hemodynamic compromise or sudden cardiac death, due to new-onset atrial or ventricular arrhythmias, complete heart block or fulminant heart failure (1-5). In particular, the occurrence of life-threatening arrhythmias during myocarditis represents a challenging clinical condition particularly in young patients. These arrhythmias are often related to myocardial damage even though several toxic or viral injuries can trigger them in patients with pre-existing arrhythomgenic focuses $(6,7)$. We present the case of a 31-year-old patient admitted to our hospital with echocardiografic and Cardiac Magnetic Resonance (CMR) signs of myocarditis complicated by ventricular tachycardia (VT) initially resolved with direct current (DC) shock. After the recurrence of VT the patient was submitted to electrophysiological study, in which the programmed ventricular stimulation induced a VT with right bundle branch block morphology and left axis deviation. Electroanatomic mapping of VT revealed the presence of a re-entrant circuit at the level of the medium segment of interventricular septum, successfully treated with transcatheter ablation without recurrences of VT.

\section{Case presentation}

A 31-year-old male was admitted to the emergency department complaining of palpitations and dizziness. The week before he had felt the same symptoms with lower intensity, regressed with rest in few minutes. The day before admission the patient had assumed a slimming product containing synephrine. Past medical history included gastroenteritis 20 days before hospital admission, with diarrhoea and heartburn, without fever, regressed after 10 days. The patient had no previous history of allergy or asthma. On admission, electrocardiogram (ECG) showed VT at high ventricular rate (280 beats/minute), with presence of pulse (Fig. 1). Blood pressure was 140/70 $\mathrm{mmHg}$, transcutaneous oxygen saturation was $98 \%$ in air. At physical examination the patient appeared oriented, mnesic in the absence of neurological deficit (Glasgow Coma Scale 15). Chest respiratory noise was present in the whole field, no rales or wheezes were present. Abdominal examination was within normal limits. Peripheral oedema or other sign or volume overload were not present. At cardiovascular examination there were no systolic or diastolic murmurs. Biomarkers of cardiac injury were in range. The patient was treated with DC shock (150 Joule) which was effective in restoring sinus rhythm. After electrical cardioversion, ECG showed slight ascendant ST segment depression in the anterior leads, mild intraventricular conduction delay. Echocardiography showed normal left ventricular diameters (left ventricular diastolic diameter: $44 \mathrm{~mm}$ ) and thicknesses, with hypokinesia of midbasal posterior and lateral wall, and ejection fraction was at the lower limits (50-55\%), with mild-to-moderate mitral regurgitation. Aortic root, left atrial and right sections diameters were within normal limits. Right ventricular function was preserved (Tricuspid Annular Plane Systolic Excursion was $25 \mathrm{~mm}$ ). There were no abnormalities of the epipericardial junction. The patient was admitted to the Cardiac Step Down Unit (CSDU) of our Institution for monitoring and further investigations: 
serological studies for cardiotropic agents and the search for autoantibodies related to immunological disorders resulted negative. Troponin I showed a slight increase soon after DC shock (maximum value: $0.52 \mathrm{ug} / \mathrm{L}$ ). Cardiac involvement of amyloidosis was excluded by means of a periumbilical fat biopsy. A diagnosis of cardiac sarcoidosis was excluded since our patients did not sufficiently met the criteria for diagnosis of clinical cardiac sarcoidosis as reported by Modified Guidelines for the Diagnosis of Cardiac Sarcoidosis from the Japanese Ministry of Health and Welfare $(8,9)$, even though we did not perform a myocardial gallium-67 scintigraphy. Moreover, in our patient no other signs suggestive for extracardiac sarcoidosis, such as hilar lymphadenopathy or interstitial lung involvement at chest X-Ray, was detected. The patient underwent CMR imaging, whose findings were suggestive for myocarditis, showing intramural late enhancement in the mid-basal septum and posterior wall and subepicardial late enhancement in anterior and lateral walls (Fig. 2 and 3). Five days after monitoring in CSDU without significant arrhythmias, the patient was transferred to the cardiology ward. One day after, a fast sustained VT was documented on cardiac monitoring, lasting 8 seconds, regressed spontaneously. The day after, another episode of VT was documented, lasting 3 minutes, associated with dizziness (Fig. 4). The patient was sent back to the CSDU and was submitted to coronary angiography, showing coronary arteries free of lesions. Given the recurrence of VT, an electrophysiological study was performed, in which the programmed ventricular stimulation induced a VT with right bundle branch block morphology and left axis deviation. Electroanatomic mapping of VT revealed the presence of a re-entrant circuit at the level of the medium interventricular septum, successfully treated with trans-catheter ablation. After the procedure, no VT resulted inducible at electrophysiological testing. The ECG monitoring in the subsequent days didn't show any complex arrhythmia except for brief runs of VT (maximum 4 beats), while CMR imaging showed a mild reduction in the myocardial inflammatory pattern. The patient was submitted to an ergometric test, conducted during therapy with verapamil, until a frequency of 155 beats/minute, which resulted negative for effort inducible arrhythmias. One month after transcatheter ablation the patient was submitted to Holter electrocardiogram monitoring showing only

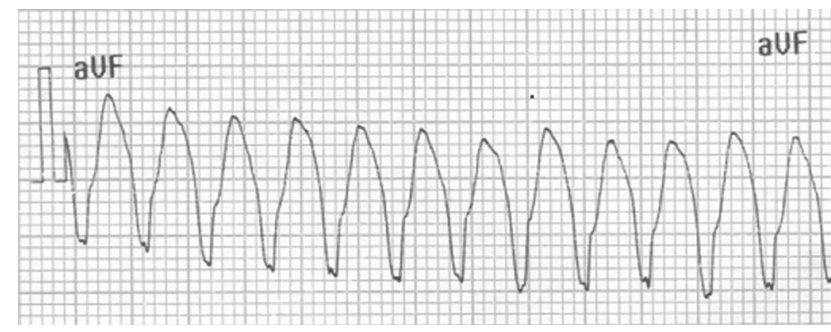

Fig. 1: Ventricular Tachycardia at presentation
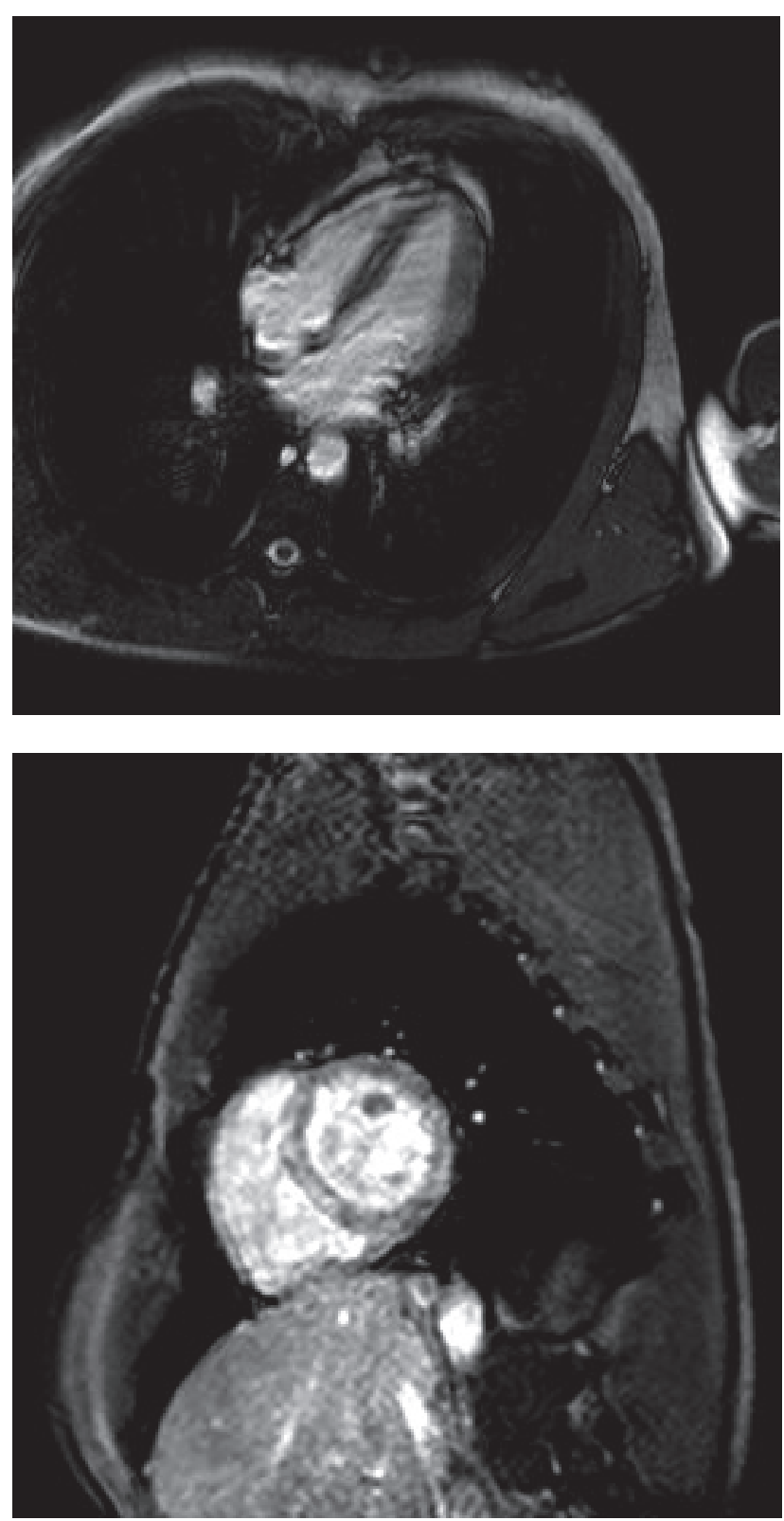

Fig. 2-3: CMR imaging showing intramural late enhancement in correspondence of the septum and subepicardial late enhancement in the lateral wall

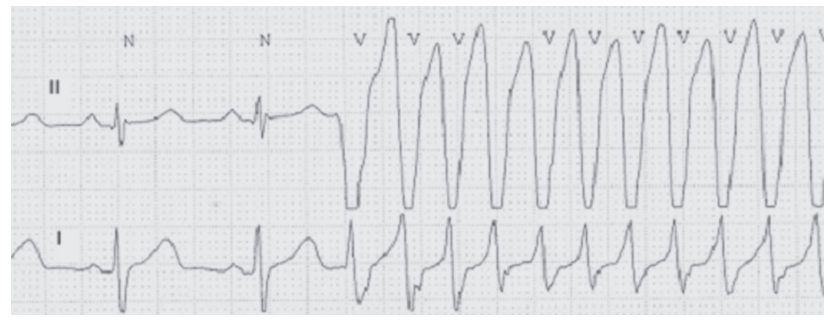

Fig. 4: Recurrence of Ventricular Tachycardia 
isolated infrequent ventricular extrasystolia during verapamil treatment, which was assumed for one month after discharge. At one year follow-up the patient was asymptomatic, echocardiogram showed hypokinesia of the posterior wall and apical posterior septum, with a preserved systolic function. A CMR was also planned, though the patient refused this examination and it was not possible to assess the evolution of myocardial inflammation.

\section{Discussion}

The clinical presentation of myocarditis can involve a wide variety of manifestations, from subclinical disease, possibly with only nonspecific electrocardiographic abnormalities and mild increase in biomarkers of myocardial damage, to acute hemodynamic compromise or sudden cardiac death, due to new-onset atrial or ventricular arrhythmias, complete heart block or fulminant heart failure $(1,2)$. Data from postmortem examinations suggest that myocarditis is the cause of sudden cardiac death in about $10 \%$ of cases (3). Among patients with suspected acute or chronic myocarditis who were screened in the European Study of Epidemiology and Treatment of Cardiac Inflammatory Diseases $72 \%$ had dyspnoea, 32\% had chest pain and $18 \%$ had arrhythmias (4); these latter are therefore a common and often the only clinical symptom in the natural course of the disease. Patients may report a viral prodrome with fever, myalgias and respiratory or gastrointestinal symptoms even if the incidence of a previous infective syndrome varies from $10 \%$ to $80 \%$ of patients with documented myocarditis (3). The wide variation in its clinical manifestations has made difficult to determine the true incidence of myocarditis. To date, the diagnosis of myocarditis can be supported by the use of imaging techniques such as CMR, echocardiography, cardiac necrosis biomarkers measurement, electrocardiographic abnormalities (3), while endomyocardial biopsy is generally reserved only for patients presenting with heart failure when a specific diagnosis is suspected that would influence therapy (5). In our patient endomyocardial biopsy was not taken into account since no sign of new-onset heart failure was detected: this procedure should have in fact been recommended in presence of unexplained, new-onset heart failure of 2 weeks' to 3 months' duration in association with a dilated ventricle and new ventricular arrhythmias. Unfortunately, without this examination, specific causes of myocarditis, such as giant-cell, eosinophilic and lymphocytic myocarditis cannot be definitively excluded. However, in our patient giant-cell myocarditis can be excluded with a high probability for the good prognosis of our patient at 1-year follow-up; eosinophilic myocarditis had a low probability because our patient had no history of allergy, asthma and no acute peripheral blood eosinophilia; lymphocytic myocarditis could not be completely excluded even though serological testing for acute infection by the three main viruses associated with this disease (cytomegalovirus, parvovirus and Coxsackie) resulted negative. On the other hand, drug induced hypersensitivity together with an underlying arrhythmogenic substrate remains a highly probable hypothesis since, even after withdrawal of synephrine, the patient continued to experience ventricular arrhythmias.

This case shows the association between acute myocarditis and recurrent VT, which is a challenging condition for physicians particularly when it occurs in young patients. The origin of these arrhythmias in this clinical setting may be due to acute myocardial damage itself or to the trigger of ventricular arrhythmias in patients with a predisposing substrate such as, in the present case, a pre-existing arrhythomgenic focus. The occurrence of ventricular arrhythmias during acute myocarditis can be triggered by fibrosis, scarring of myocardial tissue and secondary hypertrophy that can lead to the development of ectopic pacemakers, late potentials and re-entrant circuits as a result of inhomogeneous conduction of cardiac electrical impulse. Moreover, the inflammatory process within the myocytes or in the interstitium may be also involved since it can induce fluctuations in the membrane potential due to abnormal function of ion channels, in particular when located in electrically sensitive myocardial areas. Finally myocardial ischemia could be further implicated $(6,7)$. Our case suggests that the presence of recurrent ventricular arrhythmias during myocarditis could hide a pre-existing arrhythmogenic substrate. Even though we cannot exclude that the presence of an underlying arrhythmogenic focus, detected in our patient, may be an incidental finding; it could also have contributed, along with inflammation, to the recurrence of ventricular arrhythmias. In fact, idiopathic left ventricular tachycardia (fascicular VT) is usually seen in young males, between 15 and 40 years of age, with structurally normal heart, with a high prevalence in south-east Asiatic people. It is likely due to the presence of a reentrant circuit, located in the Purkinje network, in the region of the left posterior or anterior fascicle. The slow conduction zone, participating in the reentry circuit, seems to be located in a relatively wide area, from the basal to the apical left ventricular septum; in our patient it resulted in the medium segment. The clinical features of this VT include a structurally normal heart, VT with a relatively narrow QRS $(<140 \mathrm{~ms})$, a right bundle branch block in V1, left or right axis deviation, induction with atrial pacing and senisitivity to verapamil, that results effective in acute treatment, however oral verapamil shows poor efficacy in preventing VT relapses. Ablative approach is very effective for the treatment of this arrhythmia (10-11). The characteristics of ventricular arrhythmia found in our patients reflect those of fascicular tachycardia except for the absence of a structurally normal heart, thus suggesting that a myocardial insult could trigger these arrhythmia in predisposed subjects. Therefore, we suggest that patients presenting with recurrent ventricular arrhythmias and myocarditis, suspected or proven, with an otherwise structurally normal heart, should be submitted to an electrophysiological study in order to rule out the presence of arrhythmogenic 
focuses which can be treated with transcatheter ablation. Scarce data are available in the literature concerning the role of electrophysiological study in patients with myocarditis and further studies should be performed to clarify this issue. To date the common practice is to wait for myocarditis to subside by repeating another CMR and if it does, and the patient continues to have symptoms such as syncope, dizziness or even rhythm abnormalities on either holter/ event or ECG, further investigations, should be advised in the form of electrophysiological study. However in the present case, given the recurrence of VT during acute myocarditis, electrophysiological study resulted useful and allowed the identification and elimination of the arrhythmogenic focus, so helping in overcoming concerns on the possible implantation of cardioverter defibrillator, whose indications, recommended by International Guidelines, are cardiac sarcoidosis, giant cell myocarditis and Chagas disease (class IIa, LOE C) (12). However, Implantable Cardioverter-Defibrillator is not recommended in patients with acute myocarditis, since it is considered a transient or correctable arrhythmic trigger (13). Another possibility may nowadays be represented by placing an external wearable defibrillator to bridge an uncertain period of risk for recurrent ventricular arrhythmias, until the execution of a control cardiac magnetic resonance (14).

In the present case, a specific viral or autoimmune etiology at the base of myocarditis was not identified and as a further confounding factor, there remains, few days before the event, the assumption of a slimming product containing synephrine, a substance capable of inducing both ventricular arrhythmias and toxic damage to the myocardium (15). Anyway in this patient, whether myocarditis was caused by an infectious or toxic agent, the concomitant presence of recurrent ventricular arrhythmias allowed to reveal a concomitant arrhythmogenic focus by means of electrophysiological study, suggesting that this examination could be useful in the routine management of patients with recurrent VT complicating myocarditis.

\section{References}

1. Cooper LT. Myocarditis. N Engl J Med 2009; 360: 1526-38.

2. Shultz JC, Hilliard AA, Cooper LT, Rihal CS. Diagnosis and Treatment of Viral Myocarditis. Mayo Clin Proc 2009; 84: 1001-9.

3. Magnani JW, Dec GW. Myocarditis: Current Trends in Diagnosis and Treatment. Circulation 2006; 113: 876-90.

4. Hufnagel G, Pankuwei S, Ritcher A, Schönian U, Maisch B. The European Study of Epidemiology and Treatment of Cardiac Inflammatory Diseases (ESETCID): first epidemiological results. Herz 2000; 25: 279-85.

5. Jessup M, Abraham WT, Casey DE, et al. 2009 Focused Update: ACCF/AHA Guidelines for the Diagnosis and Management of Heart Failure in Adults: A Report of the American College of Cardiology Foundation/American Heart Association Task Force on Practice Guidelines: Developed in Collaboration With the International Society for Heart and Lung Transpalntation. Circulation 2009; 119: 1977-2016.

6. Chevalier P, Scridon A. Ventricular arrhythmias complicating acute myocarditis. E-journal of the ESC Council for Cardiology Practice 2011; 9: 26.

7. Klein RM, Vester EG, Brehm MU, et al. Inflammation of the myocardium as an arrhythmia trigger. Z Kardiol 2000; 89: 24-35.

8. Hiraga H, Yuwai K, Hiroe M. Guideline for diagnosis of cardiac sarcoidosis: study report on diffuse pulmonary disease from the Japanese Ministry of Health and Welfare (in Japanese). Japanese Ministry of Health and Welfare 1993; 6: 23-24.

9. Diagnostic standard and guidelines for sarcoidosis. Jpn J Sarcoidosis and Granulomatous Disorders 2007; 27: 89-102.

10. Chiarandà G, Di Guardo G, Gulizia M, Lazzaro A, RegoloT. Fascicular Ventricular Tachycardia. Ital Heat J Suppl 2001; 2: 1181-6.

11. Nogami A. Idiophatic left ventricular tachycardia: assessment and treatment. Card Electrophysiol Rev 2002; 6: 448-57.

12. Epstein AE, Di Marco JP, Ellenbogen KA, et al. ACC/AHA/HRS 2008 Guidelines for Device-Based Therapy of Cardiac Rhythm Abnormalities: A Report of the American College of Cardiology/American Heart Association Task Force on Practice Guidelines (Writing Committee to Revise the ACC/AHA/NASPE 2002 Guideline Update for Implantation of Cardiac Pacemakers and Antiarrhythmia Devices): Developed in Collaboration With the American Association for Thoracic Surgery and Society of Thoracic Surgeons. Circulation 2008; 117: e350-e408.

13. Zipes DP, Camm AJ, Borggrefe M, et al. ACC/AHA/ESC 2006 Guidelines for management of patients with ventricular arrhythmias and the prevention of sudden cardiac death. A report of the American College of Cardiology/American Heart Association Task Force and the European Society of Cardiology Committee for Practice Guidelines (Writing Committee to Develop Gudelines for Management of Patients With Ventricular Arrhythmias and the Prevention of Sudden Cardiac Death) Developed in Collaboration With the European Heart Rhythm Association and the Heart Rhythm Society. Europace 2006; 8: 746-837.

14. Prochnau D, Surber R, Kuehnert H, Heinke M, Klein HU, Figulla HR. Succesfull use of a wearable cardioverter-defibrillator in myocarditis with normal ejection fraction. Clin Res Cardiol 2010; 99: 129-31.

15. Stephensen TA, Sarlay R. Ventricular Fibrillation Associated With Use of Synephrine Containing Dietary Supplement. Mil Med 2009; 174: 1313-9.

Received: 03/01/2012

Accepted in revised form: 11/04/2012

\section{Corresponding author:}

Emanuele Cecchi, MD, Dipartimento del Cuore e dei Vasi, Azienda Ospedaliero-Universitaria Careggi, Viale Morgagni 85, 50134 Florence Italy; e-mail: emanuelececchi@virgilio.it 